\title{
Colossal Proximity Effect in a Superconducting Triplet Spin Valve Based on the Half-Metallic Ferromagnet $\mathrm{CrO}_{2}$
}

\author{
A. Singh, S. Voltan, K. Lahabi, and J. Aarts \\ Kamerlingh Onnes-Huygens Laboratory, Leiden University, P.O. Box 9504, \\ 2300 RA Leiden, The Netherlands \\ (Received 25 November 2014; published 26 May 2015)
}

\begin{abstract}
Combining superconductors $(S)$ and ferromagnets $(F)$ offers the opportunity to create a new class of superconducting spintronic devices. In particular, the $S / F$ interface can be specifically engineered to convert singlet Cooper pairs to spin-polarized triplet Cooper pairs. The efficiency of this process can be studied using a so-called triplet spin valve (TSV), which is composed of two $F$ layers and a $S$ layer. When the magnetizations in the two $F$ layers are not collinear, singlet pairs are drained from the $S$ layer, and triplet generation is signaled by a decrease of the critical temperature $T_{c}$. Here, we build highly efficient TSVs using a $100 \%$ spin-polarized half-metallic ferromagnet, $\mathrm{CrO}_{2}$. The application of out-of-plane magnetic fields results in an extremely strong suppression of $T_{c}$, by well over a Kelvin. The observed effect is an order of magnitude larger than previous studies on TSVs with standard ferromagnets. Furthermore, we clearly demonstrate that this triplet proximity effect is strongly dependent on the transparency and spin activity of the interface. Our results are particularly important in view of the growing interest in generating long-range triplet supercurrents for dissipationless spintronics.
\end{abstract}

DOI: 10.1103/PhysRevX.5.021019

Ferromagnets $(F)$ can sustain supercurrents through the formation of equal spin-triplet Cooper pairs and the mechanism of odd-frequency pairing. Since such pairs are not broken by the exchange energy of the ferromagnet, superconducting triplet correlations are long ranged and spin polarized, with promises for superconducting spintronics devices [1-3]. In superconductor/ferromagnet $(S / F)$ hybrids, the spin-polarized triplet correlations can be generated by converting Cooper pairs from the singlet to the triplet state via spin mixing and spin rotation at the $S-F$ interface, which requires the presence of magnetic inhomogeneity [4-8]. Recently, it was shown that long-range supercurrents could be engineered in $S / F / S$ Josephson junctions by inserting an extra ferromagnetic layer between the superconductor and the central $F$ layer [9-12]. Still, quantitative understanding of the conversion process is mostly lacking since the spin activity of the interface is not a measurable parameter. Absolute values of the supercurrent are not easily predictable, which was illustrated clearly in recent work of Klose et al. [13], where supercurrents in a Co-based Josephson junction could be increased more than an order of magnitude by manipulating the magnetization directions of $F_{1}$ and $F_{2}$. For acquiring

Published by the American Physical Society under the terms of the Creative Commons Attribution 3.0 License. Further distribution of this work must maintain attribution to the author(s) and the published article's title, journal citation, and DOI.
Subject Areas: Condensed Matter Physics, Magnetism, Superconductivity

such understanding, a Josephson junction has the disadvantage that it contains two sets of interfaces, which may not have the same amount of spin activity or even transparency. In this sense, a triplet spin valve (TSV), pictorially sketched in Fig. 1(a), is a simpler device. It can be thought of as half of the Josephson junction, utilizing the same layer package $S / F_{1} / N / F_{2}$. The $S$ layer is chosen not too thick, so that drainage of Cooper pairs through triplet conversion is reflected in the change of $T_{c}$ of the stack, $F_{1}$ is thin in order to take part in the spin mixing, but not to break Cooper pairs, and $F_{2}$ is the drainage layer, which can be infinitely thick. By changing the relative magnetization directions of $F_{1}$ and $F_{2}$, the triplet pair generation is varied and thereby the amount of singlet pairs that is converted, making the operation a field-controlled proximity effect. When $F_{1}$ and $F_{2}$ are orthogonal, triplet generation is maximum and $T_{c}$ should be minimum. The performance of a TSV can be gauged by the extent to which $T_{c}$ decreases, and, as we show below, interface transparency can also be explicitly addressed.

There are several recent experimental results on TSVs, which use standard ferromagnets $(\mathrm{Fe}, \mathrm{Co}, \mathrm{Ni})$ and their alloys as spin mixers and drainage layers [14-18]. In all cases, magnetic anisotropy or an antiferromagnetic pinning layer was used to reliably control the relative magnetization directions, always in the plane of the films. The maximum suppression of $T_{c}$ achieved in such devices ranged from $120 \mathrm{mK}$ (for a thin ballistic Co drainage layer) [15] to $20 \mathrm{mK}$ (for diffusive TSVs) $[14,17,18]$. Our experiments 
(a)

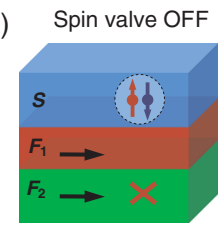

(b) $\square \mathrm{CrO}_{2} \quad \square \mathrm{MoGe} / \mathrm{Ni} / \mathrm{Cu}$

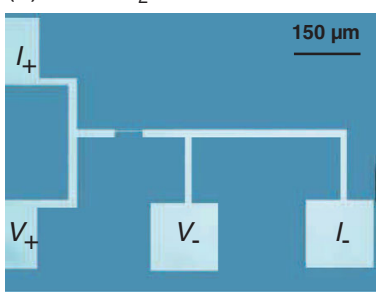

Spin valve ON

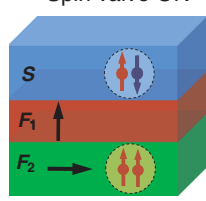

(c)

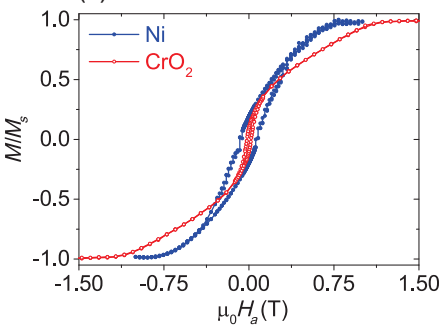

FIG. 1. Device structure and magnetic characterization. (a) Working principle of a triplet spin valve (TSV) with a half-metallic ferromagnet; the TSV is off (on) when the magnetizations of $F_{1}$ and $F_{2}$ are collinear (noncollinear), with a maximum effect when they are orthogonal. (b) Optical micrograph of a typical TSV where a $\operatorname{MoGe}\left(d_{s}\right) / \mathrm{Ni}(1.5 \mathrm{~nm}) /$ $\mathrm{Cu}(5 \mathrm{~nm})$ trilayer bridge of $10 \mu \mathrm{m}$ width is patterned on a $100 \mathrm{~nm}$ thin film of $\mathrm{CrO}_{2}$. (c) Magnetization hysteresis loops for $\mathrm{CrO}_{2}(100 \mathrm{~nm})$ and a multilayer $[\mathrm{Ni}(1.5 \mathrm{~nm}) / \mathrm{Cu}(10 \mathrm{~nm})]_{11} /$ $\mathrm{Ni}(3 \mathrm{~nm}) / \mathrm{Cu}(10 \mathrm{~nm})$ measured with the magnetic field perpendicular to the sample plane. The magnetization $M$ is normalixed on the saturation magnetization $M_{s}$, which is $6.8 \times 10^{5} \mathrm{~A} / \mathrm{m}$ for the $\mathrm{CrO}_{2}$ film and $2.2 \times 10^{5} \mathrm{~A} / \mathrm{m}$ for the $\mathrm{Cu} / \mathrm{Ni}$ multilayer.

are different in two important aspects. One is the use of the half-metallic ferromagnet $\mathrm{CrO}_{2}$ as the drainage layer. The other is that we vary the field from in plane to out of plane. Unlike in-plane rotation of the field, out-of-plane rotation changes the critical field of the superconductor itself. We, therefore, account for this by comparing our TSV with stacks where one of the $F$ layers is absent, as well as with the simple $S$ layer. In this way, we find that spin valve effects are present up to fields of Teslas, and they are remarkably large, with a suppression in $T_{c}$ as high as $1.8 \mathrm{~K}$ in $0.5 \mathrm{~T}$. The origin of this significant variation probably lies in the fact that $\mathrm{CrO}_{2}$ is $100 \%$ spin polarized and strongly supports triplet correlations.

Our TSV is made of amorphous MoGe, $\mathrm{Ni}, \mathrm{Cu}$, and $\mathrm{CrO}_{2}$ as the $S, F_{1}, N$, and $F_{2}$ layers, respectively. The $\mathrm{Cu}$ layer is required to magnetically decouple the mixer and drainage layers. The investigated devices consist of a 100-nm-thick $\mathrm{CrO}_{2}$ film grown on a $\mathrm{TiO}_{2}$ substrate by chemical vapor deposition, on top of which a $10-\mu \mathrm{m}$-wide $\mathrm{MoGe} / \mathrm{Ni} / \mathrm{Cu}$ trilayer bridge is deposited using sputtering and lift-off [19]. Prior to the trilayer deposition, the top surface of $\mathrm{CrO}_{2}$ is cleaned with an argon ion plasma to remove the thin insulating $\mathrm{Cr}_{2} \mathrm{O}_{3}$ barrier that is prone to form at the end of the deposition process. An optical micrograph of one such device is shown in Fig. 1(b). In this geometry, similar to the Josephson junction devices

$[12,19,20]$, the current is confined to the bridge only in the superconducting state. To characterize the magnetic properties of $F_{1,2}$ layers, their hysteresis loops (magnetization $M$ versus applied field $H_{a}$ ) are measured using SQUID magnetometry in the out-of-plane configuration. Instead of a single $\mathrm{Ni}$ layer of $1.5 \mathrm{~nm}$, we use a multilayer $[\mathrm{Ni}(1.5) / \mathrm{Cu}(10)]_{11} / \mathrm{Ni}(3) / \mathrm{Cu}(10)$ in order to boost the signal. The data are given in Fig. 1(c) and show that in both layers the rotation of the magnetization requires a field of order of a Tesla.

Electrical measurements are performed in a four-probe configuration in a physical properties measurement systems. For angle-resolved magnetotransport measurements, the magnetic field $\left(H_{a}\right)$ is rotated in a plane normal to the sample. In this geometry, when $\theta=0^{\circ}$ the field is aligned with the current density $(j)$, and $\theta=90^{\circ}$ corresponds to the out-of-plane applied field as outlined in Fig. 2(a). Figure 2(b) (upper panels) shows $R(T)$ curves at different angles of the magnetic field for two TSVs consisting of $\operatorname{MoGe}\left(d_{S}\right) / \mathrm{Ni}(1.5 \mathrm{~nm}) / \mathrm{Cu}(5 \mathrm{~nm}) / \mathrm{CrO}_{2}(100 \mathrm{~nm})$, with two different values of the MoGe thickness $d_{S}=25 \mathrm{~nm}$ (called TSV25a) and $d_{S}=50 \mathrm{~nm}$ (called TSV50a), and for fixed magnetic fields of 0.25 and $0.5 \mathrm{~T}$. We extract an operational parameter $T_{50 \%}$ (for a discussion of this choice, see the Supplemental Material [21]), which is the temperature where the resistance has decreased to $50 \%$ of the normal resistance value. The variation of $T_{50 \%}$ with $\theta$ is called $\delta T_{50 \%}=T_{50 \%}\left(0^{\circ}\right)-T_{50 \%}(\theta)$. The lower panels show $\delta T_{50 \%}$ as a function of $\theta$, and the curves clearly exhibit a maximum when the field is normal to the plane. Further points to note are (i) the large values of the change, of about 550 and $650 \mathrm{mK}$ for TSV50a in 0.25 and $0.5 \mathrm{~T}$, respectively, and $750 \mathrm{mK}$ for TSV25a in $0.5 \mathrm{~T}$, (ii) the significantly larger value of the normal state resistance for TSV25a, and (iii) the sharp peak in resistance, which in the parallel field occurs at the onset of superconductivity and which smears out and disappears when rotating the field.

In order to discuss the first point, we have to put the data in perspective. The superconductor itself will show a $T_{50 \%}(\theta)$ variation, because the transition in the parallel field is due to the onset of surface superconductivity, which is at a higher field and temperature than the transition in the perpendicular critical field. The change from surface to bulk effects also raises concerns about going from a vortexfree configuration to one where vortex flow may play a role. These issues are resolved by a straightforward comparison with the behavior of a single MoGe layer, for which we take a thickness of $50 \mathrm{~nm}$. Stray fields of mixer and drainage layer may also play a role, and therefore we compare with devices of $\operatorname{MoGe}(50 \mathrm{~nm}) / \mathrm{Ni}(1.5 \mathrm{~nm}) / \mathrm{Cu}(5 \mathrm{~nm})$ and $\operatorname{MoGe}(50 \mathrm{~nm}) / \mathrm{Cu}(5 \mathrm{~nm}) / \mathrm{CrO}_{2}(100 \mathrm{~nm})$ as well. Figure 3(a) shows the transition curves of these devices at $0.25 \mathrm{~T}$ for in-plane and out-of-plane configurations. All have comparable $\delta T_{50 \%}$. In Fig. 3(b), values of $\delta T_{50 \%}$ for the different data sets are compared, again at a field of 


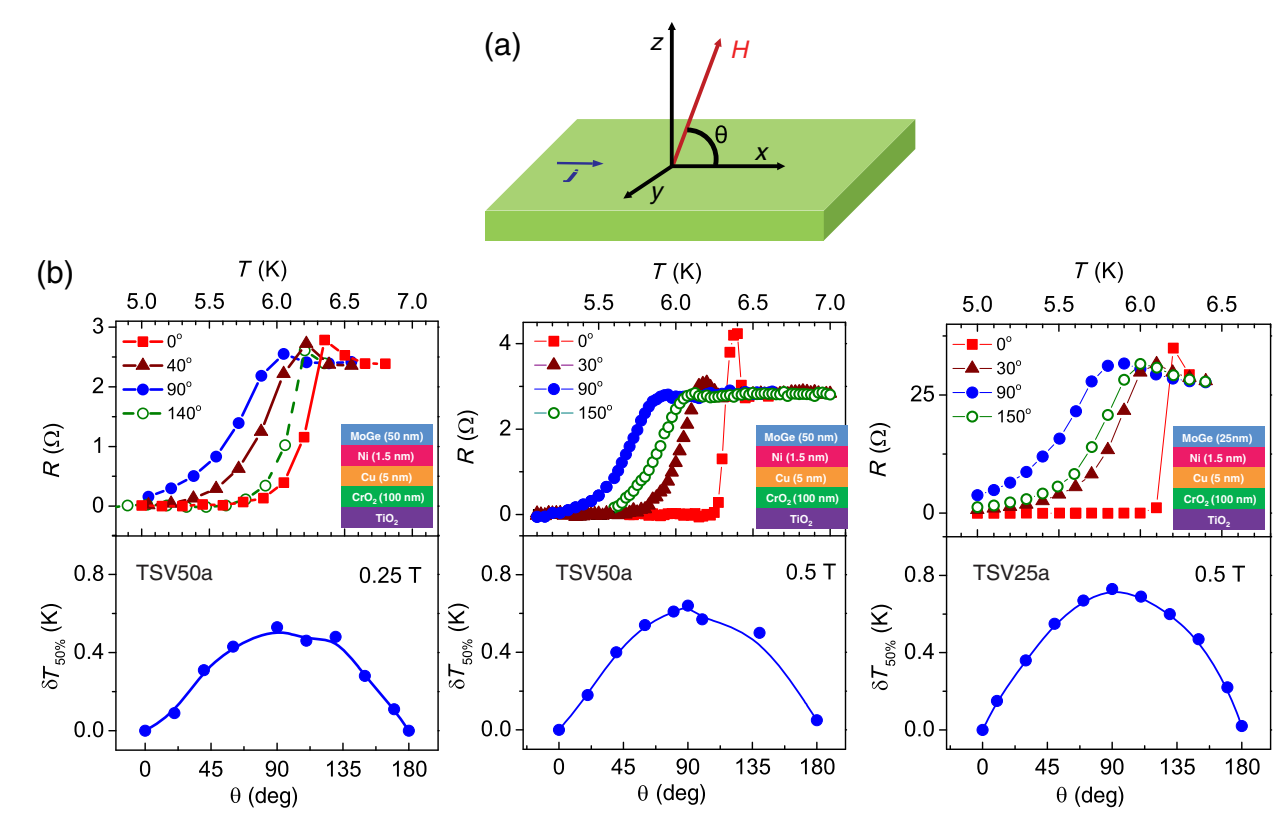

FIG. 2. Colossal triplet spin valve effect. (a) Coordinate system used in angle-dependent magnetotransport measurements, showing the direction of the current $j$, the applied field $H_{a}$, and the angle $\theta$ between them. (b) Spin valve effect in the two spin valves $\operatorname{MoGe}\left(d_{s}\right) / \mathrm{Ni}(1.5 \mathrm{~nm}) / \mathrm{Cu}(5 \mathrm{~nm}) / \mathrm{CrO}_{2}(100 \mathrm{~nm})$ with $d_{s}=5 \mathrm{~nm}\left(\mathrm{TSV} 50 \mathrm{a}\right.$, left and middle) and $d_{s}=25 \mathrm{~nm}(\mathrm{TSV} 25 \mathrm{a}$, right). Upper panels: Resistive transitions for different $\theta$ as indicated. Lower panels: Variation of $\delta T_{50 \%}=T_{50 \%},\left(0^{\circ}\right)-T_{50 \%},(\theta)$ as a function of $\theta$ at $0.25 \mathrm{~T}$ (TSV50a) and $0.5 \mathrm{~T}$ (TSV50a, TSV25a), where $T_{50 \%}$ is the temperature where the normal state resistance has decreased by $50 \%$. Note that a peak appears in the transition curves for measurements at $\theta=0^{\circ}$.

$0.25 \mathrm{~T}$. It can be clearly seen that the variation in the TSV is significantly larger than in the other devices. Figure 3(c) shows the variation of $\delta T_{50 \%, \max }=T_{50 \%}\left(0^{\circ}\right)-T_{50 \%}\left(90^{\circ}\right)$ as a function of the applied field for an isolated MoGe film and a TSV with a $50 \mathrm{~nm}$ MoGe layer called TSV50b.
In both cases, $\delta T_{50 \%, \text { max }}$ increases monotonically with the magnetic field up to 2 T. The shaded area in Fig. 3(c) corresponds solely to the effect of triplet generation, which can suppress $\delta T_{50 \% \text {,max }}$ by as much as $800 \mathrm{mK}$. We find TSV effects over a wide range of magnetic fields. This was
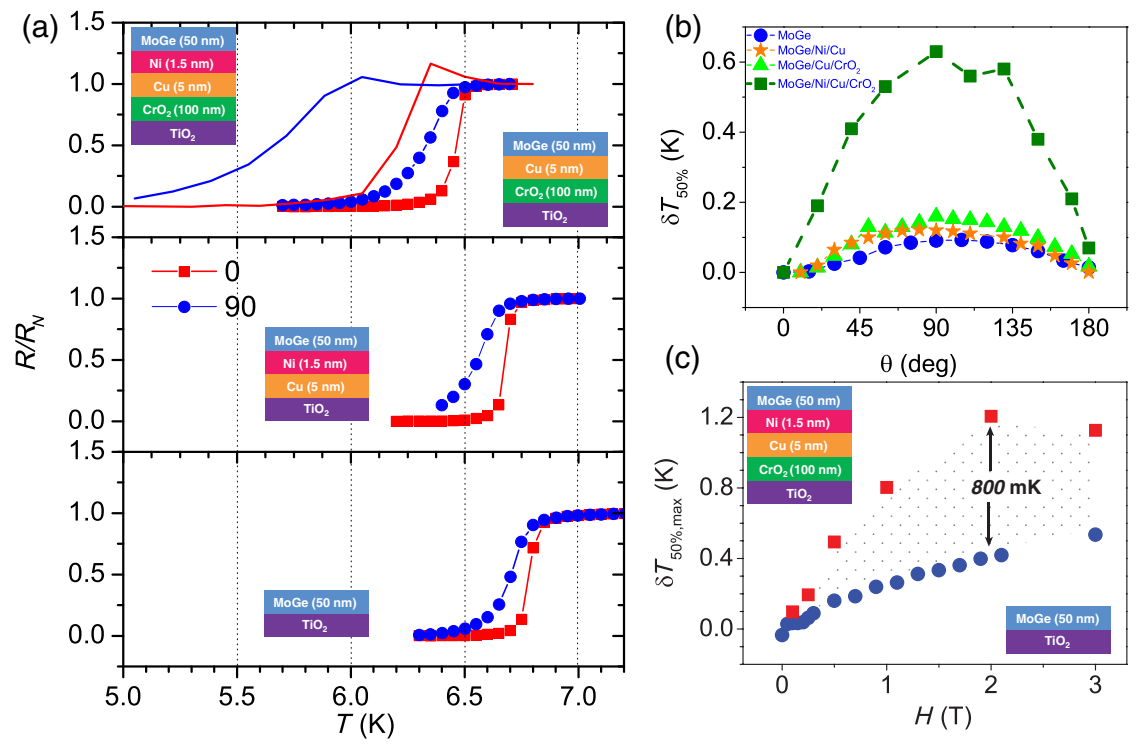

FIG. 3. Non-triplet-generating layer combinations. (a) Transition curves for $\mathrm{MoGe}(50 \mathrm{~nm}) / \mathrm{Cu}(5 \mathrm{~nm}) / \mathrm{CrO} 2(100 \mathrm{~nm})$ (top), $\operatorname{MoGe}(50 \mathrm{~nm}) / \mathrm{Ni}(1.5 \mathrm{~nm}) / \mathrm{Cu}(5 \mathrm{~nm})$ (middle), and $\operatorname{MoGe}(50 \mathrm{~nm})$ (bottom) for $\theta=0^{\circ}$ and $\theta=90^{\circ}$ at $0.25 \mathrm{~T}$. The top panel also shows the results of the spin valve device TSV50a as drawn lines. (b) $\delta T_{50 \%}$ as a function of $\theta$ for these layered devices and for TSV50a at $0.25 \mathrm{~T}$. (c) Variation of $T_{50 \%, \max }=T_{50 \%},\left(0^{\circ}\right)-T_{50 \%},\left(90^{\circ}\right)$ as a function of applied field for MoGe(50) and TSV50b. 
not the case for previous TSVs measured in an in-plane configuration, where the maximum field of operation was limited to $0.2-0.3 \mathrm{~T}$ [18]. Robust proximity effects were also observed in $\mathrm{CrO}_{2}$-based Josephson junctions $[12,19,20]$, where critical currents in various configurations were observed up to the Tesla range. It is slightly puzzling that $\delta T_{50 \% \text {,max }}$ continues to increase well above the fields where saturation of both mixer and drainage layer have been achieved and both magnets are assumed to be collinear. We believe that this may be caused by the presence of noncollinear magnetic moments pinned at the $\mathrm{CrO}_{2}$ interface. Noting that the difference between the TSV and the other stacks lies only in the insertion of an extra $1.5 \mathrm{~nm}$ layer of $\mathrm{Ni}$, it seems reasonable to conclude that, just as in the case of the Josephson junctions, the $\mathrm{Ni}$ layer is instrumental in generating triplets. They are very efficiently drained by the $\mathrm{CrO}_{2}$ layer, which leads to the observed large spin valve effects.

Turning to the larger value of the normal state resistance $R_{N}$ of TSV25a, this can be used to probe the effects of the bare interface transparency, which is a critical parameter in determining the strength of proximity effect, and much studied in $S / N$ and $S / F$ hybrids [22,23]. In our devices, the transparency of the interface between the $\mathrm{CrO}_{2}$ film and the $\mathrm{Cu} / \mathrm{Ni} / \mathrm{MoGe}$ stack is controlled by the argon etching of the $\mathrm{CrO}_{2}$ surface prior to the deposition of the stack. The etching is a critical step in the fabrication, due to the fact that underetching results in only partial removal of an unwanted $\mathrm{Cr}_{2} \mathrm{O}_{3}$ layer while overetching induces disorder at the surface of $\mathrm{CrO}_{2}$.

We take advantage of this by making different devices on the same $\mathrm{CrO}_{2}$ film using different etch times. For this the film is covered with resist, a lift-off structure is written, the $\mathrm{CrO}_{2}$ surface is etched for a certain amount of time, and the stack is deposited. This process is repeated with different etch times. The transparency has a direct influence on the normal resistance of the device, which in essence consists of a top $N$ layer (MoGe) of high resistance and a bottom $F$ layer $\left(\mathrm{CrO}_{2}\right)$ of low resistance, with an interface resistance $R_{B}$ in between. With contacts on top, $R_{B}$ is in series with the low-resistance bottom layer and its measurable influence on $R_{N}$ allows $R_{N}$ to be used as a parameter for the interface transparency. The details are given in the Supplemental Material [21]. In Fig. 4, we plot $\delta T_{50 \% \text {,max }}$ against $1 / R_{N}$ for both sets of devices measured in $0.5 \mathrm{~T}$ (blue triangles and green circles) and a set TSV50 measured in $0.25 \mathrm{~T}$ (red squares). The devices TSV50a,b and TSV25a are plotted with special (open) symbols. The performance of all TSVs increases monotonically with decreasing $R_{N}$ and increasing barrier transparency. Interface transparency also offers a natural explanation for the fact that TSV25a devices with higher $R_{N}$ exhibit an effect comparable to the set TSV50. According to basic proximity effect theory, the thinner layer should show a stronger effect upon Cooper pair depletion, but as can be

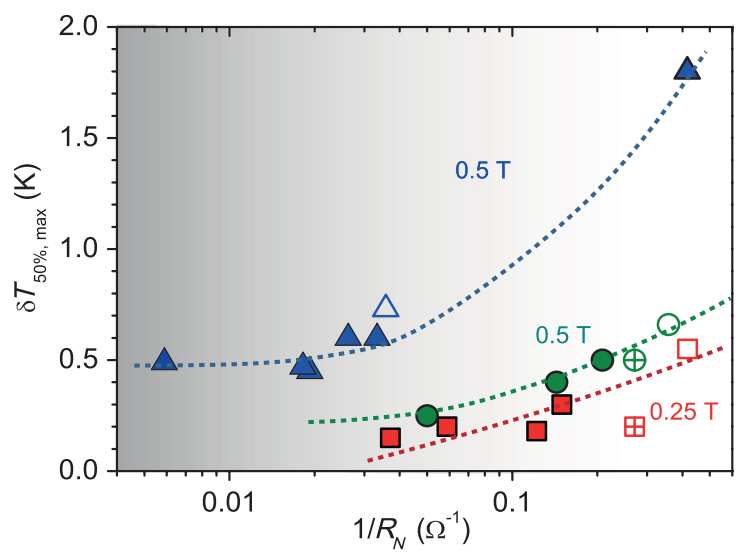

FIG. 4. Dependence of TSV effect on interface transparency. Variation of $T_{50 \% \text {,max }}$ plotted against the inverse of the normal state resistance $R_{N}$ (interface transparency), for spin valve devices with different interfaces between the $\mathrm{CrO}_{2}$ layer and the $\mathrm{MoGe} / \mathrm{Ni} / \mathrm{Cu}$ stack. Blue triangles: TSV25 $\left(d_{S}=25 \mathrm{~nm}\right)$, measured at $0.5 \mathrm{~T}$; open symbol: TSV25a. Green circles (red squares): TSV50 $\left(d_{S}=50 \mathrm{~nm}\right)$, measured at $0.5 \mathrm{~T}(0.25 \mathrm{~T})$. Symbols with + are device TSV50a, the open green circle (red square) is TSV50b.

seen in Fig. 4, this is counteracted by the lower interface transparency. In this respect, it should also be remarked that the set TSV50 is surprisingly efficient when taking into account that the $S$-layer thickness is about 10 times the superconducting coherence length $\xi_{S}$, which for MoGe is about $5 \mathrm{~nm}$ [24]. This again appears to be a consequence of the $100 \%$ spin-polarized ferromagnet.

The final striking feature in our results is the observed characteristic peak in the transition curve of a TSV at a finite in-plane field, which disappears when the field is rotated out of the plane [see Fig. 2(b)]. As we show in Fig. 5(a) for TSV50a, the peak is not present in zero field, but then gradually appears at fields around $0.2 \mathrm{~T}$, a behavior that appears consistently in all of our devices. We believe that this effect arises due to the normal reflection of equal spin-triplet Cooper pairs at the half-metallic boundary. As pictorially shown in Fig. 5(b), we assume that $m_{s}=0$ singlets are converted into $m_{s}=0$ triplets in the $\mathrm{Ni} / \mathrm{Cu}$ sandwich, but that a triplet $m_{s}=1$ quantization axis is provided by the misaligned moments, which could be called a $F^{\prime}$ layer. When these $m_{s}=1$ triplets encounter the $\mathrm{CrO}_{2}$ bulk, they will be partly transmitted, but also partly reflected. The latter may result in the breaking of the pair on the $\mathrm{MoGe} / \mathrm{Cu} / \mathrm{Ni}$ side of the stack, resulting in quasiparticles with the same spin. This spin accumulation raises the spin chemical potential $\left(\Delta \mu=\mu_{\uparrow}-\mu_{\downarrow}\right)$ and results in additional spin contact resistance, which manifests itself as the observed peak at the onset of the superconducting transition. Typically, the spin accumulation at the $S F$ interface is quantified by excess resistance, expressed as $\Delta R=\left[P^{2} /\left(1-P^{2}\right)\right]\left(\rho l_{s d} / A\right)$, where $P, \rho$, and $l_{s d}$ are the spin polarization, resistivity, and the spin diffusion length 
(a)
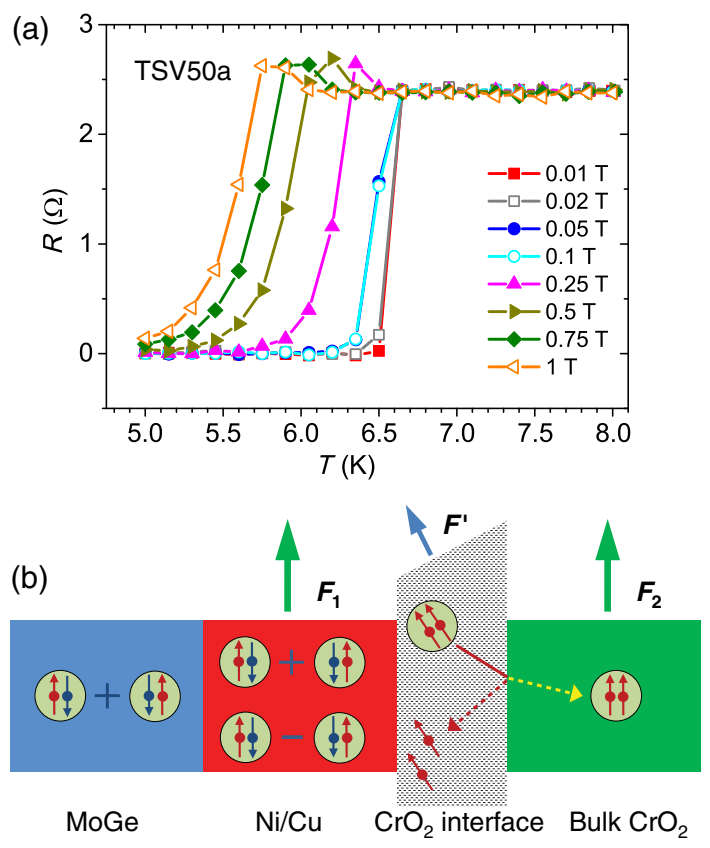

FIG. 5. Normal reflection of triplet Cooper pairs. (a) Resistive transitions of a spin valve device TSV50a, for different values of the in-plane field between 0 and $1 \mathrm{~T}$. (b) Pictorial representation of the effect of an extra ferromagnetic layer $F^{\prime}$ between the mixer layer $F_{1}$ and the drainage layer $F_{2}$ on the generation of triplet pairs. The green arrows represent magnetization directions of $F_{1,2}$ (in plane); the blue arrow indicates an interface layer $F^{\prime}$ with magnetization direction out of plane.

of the ferromagnet, respectively, and $A$ is the area of the $F / S$ junction [25]. This expression cannot be used to quantify $\Delta R$ for a half-metal as it diverges for $P=1$, but it is clear that for half-metals with $P$ close to 1 , the spin accumulation can be considerably larger than in other ferromagnets.

Spin accumulation leads to excess resistance, but that accumulation would occur is nontrivial. The zero-field state can be supposed to generate triplets since the domain state of both ferromagnets can be considered as noncollinear. Applying an in-plane field makes the $F_{1,2}$ magnetizations more collinear, but if the $F^{\prime}$ magnetization has a component perpendicular to the interface, the triplet magnetization axis would indeed be different from the bulk. In the same vein, the effect would be less for the out-of-plane configuration, in which $F^{\prime}$ and $F_{2}$ are becoming more collinear. Theoretical modeling will be needed to investigate this scenario.

To summarize, we demonstrate a triplet spin valve using a $100 \%$ spin-polarized ferromagnet. By changing the field from in plane to out of plane, we find very large effects occurring up to magnetic fields of $2 \mathrm{~T}$. We also show that the interface transparency between the bulk magnet and the triplet-generating stack has a decided effect on the efficiency of the TSV. Finally, a characteristic peak in the transition curve of the TSV with the field in plane is explained in terms of spin accumulation caused by equalspin Cooper pair breaking. We suggest that TSVs, in particular those based on half-metals, are good model systems for a systematic study of the parameters that are relevant for triplet generation. We believe that this work will motivate the development of much needed theoretical formalism of TSVs based on half-metals.

We gratefully acknowledge technical support from M. B.S. Hesselberth and D. Boltje, discussions with S. Bergeret, and assistance from S. Anwar in the initial stage of film growth. This work is part of the research program of the Foundation for Fundamental Research on Matter (FOM), which is part of the Netherlands Organisation for Scientific Research (NWO). It was supported by a grant from the Leiden-Delft Consortium "NanoFront" and by COST action MP1201.

[1] Y. V. Fominov, A. A. Golubov, T. Y. Karminskaya, M. Y. Kupriyanov, R. G. Deminov, and L. R. Tagirov, Superconducting Triplet Spin Valve, JETP Lett. 91, 308 (2010).

[2] A. K. Feofanov et al., Implementation of Superconductor/ Ferromagnet/Superconductor $\pi$-Shifters in Superconducting Digital and Quantum Circuits, Nat. Phys. 6, 593 (2010).

[3] H. Enoksen, J. Linder, and A. Sudbo, Pressure-Induced 0- $\pi$ Transitions and Supercurrent Crossover in Antiferromagnetic Weak Links, Phys. Rev. B 88, 214512 (2013).

[4] F. S. Bergeret, A. F. Volkov, and K. B. Efetov, Long-Range Proximity Effects in Superconductor-Ferromagnet Structures, Phys. Rev. Lett. 86, 4096 (2001).

[5] A. Kadigrobov, R. I. Shekhter, and M. Jonson, Quantum Spin Fluctuations as a Source of Long-Range Proximity Effects in Diffusive Ferromagnet-Superconductor Structures, Europhys. Lett. 54, 394 (2001).

[6] F. S. Bergeret, A. F. Volkov, and K. B. Efetov, Manifestation of Triplet Superconductivity in Superconductor-Ferromagnet Structures, Phys. Rev. B 68, 064513 (2003).

[7] M. Houzet and A. I. Buzdin, Long Range Triplet Josephson Effect through a Ferromagnetic Trilayer, Phys. Rev. B 76, 060504(R) (2007).

[8] M. Eschrig and T. Lofwänder, Triplet Supercurrents in Clean and Disordered Half-Metallic Ferromagnets, Nat. Phys. 4, 138 (2008).

[9] T. S. Khaire, M. A. Khasawneh, W. P. Pratt, and N. O. Birge, Observation of Spin-Triplet Superconductivity in Co-Based Josephson Junctions, Phys. Rev. Lett. 104, 137002 (2010).

[10] J. W. A. Robinson, J. D. S. Witt, and M. G. Blamire, Controlled Injection of Spin-Triplet Supercurrents into a Strong Ferromagnet, Science 329, 59 (2010).

[11] M. A. Khasawneh, T. S. Khaire, C. Klose, W. P. Pratt, Jr., and N.O. Birge, Spin-triplet supercurrent in Co-based Josephson junctions, Supercond. Sci. Technol. 24, 024005 (2011).

[12] M. S. Anwar, M. Veldhorst, A. Brinkman, and J. Aarts, Long Range Supercurrents in Ferromagnetic $\mathrm{CrO}_{2}$ Using a Multilayer Contact Structure, Appl. Phys. Lett. 100, 052602 (2012). 
[13] C. Klose, T. S. Khaire, Y. Wang, W. P. Pratt, N. O. Birge, B. J. McMorran, T. P. Ginley, J. A. Borchers, B. J. Kirby, B. B. Maranville, and J. Unguris, Optimization of Spin-Triplet Supercurrent in Ferromagnetic Josephson Junctions, Phys. Rev. Lett. 108, 127002 (2012).

[14] P. V. Leksin, N. N. Garif'yanov, I. A. Garifullin, Y. V. Fominov, J. Schumann, Y. Krupskaya, V. Kataev, O. G. Schmidt, and B. Büchner, Evidence for Triplet Superconductivity in a Superconductor-Ferromagnet Spin Valve, Phys. Rev. Lett. 109, 057005 (2012).

[15] X. L. Wang, A. Di Bernardo, N. Banerjee, A. Wells, F. S. Bergeret, M. G. Blamire, and J. W. A. Robinson, Giant Triplet Proximity Effect in Superconducting Pseudo Spin Valves with Engineered Anisotropy, Phys. Rev. B 89, 140508(R) (2014).

[16] N. Banerjee, C. B. Smiet, R. G. J. Smits, A. Ozaeta, F. S. Bergeret, M. G. Blamire, and J. W. A. Robinson, Evidence for Spin Selectivity of Triplet Pairs in Superconducting Spin Valves, Nat. Commun. 5, 048 (2014).

[17] A. J. Jara, C. Safranski, I. N. Krivorotov, C.-T. Wu, A. N. Malmi-Kakkada, O. T. Valls, and K. Halterman, Angular Dependence of Superconductivity in Superconductor/ Spin-Valve Heterostructures, Phys. Rev. B 89, 184502 (2014).

[18] M. G. Flokstra et al., Controlled Suppression of Superconductivity by the Generation of Polarized Cooper Pairs in Spin Valve Structures, Phys. Rev. B 91, 060501 (2015).
[19] M. S. Anwar, F. Czeschka, M. Hesselberth, M. Porcu, and J. Aarts, Long-Range Supercurrents through Half-Metallic Ferromagnetic $\mathrm{CrO}_{2}$, Phys. Rev. B 82, 100501(R) (2010).

[20] R. Keizer, S. T. B. Gönnenwein, T. M. Klapwijk, G. Miao, G. Xiao, and A. Gupta, A Spin Triplet Supercurrent through the Half-Metallic Ferromagnet $\mathrm{CrO}_{2}$, Nature (London) 439, 825 (2006).

[21] See Supplemental Material at http://link.aps.org/ supplemental/10.1103/PhysRevX.5.021019 for comments on sample preparation, the interface transparency, the low field performance of the TSVs and the use of the parameter $T_{50 \%}$.

[22] Y. V. Fominov, N. M. Chtchelkatchev, and A. A. Golubov, Nonmonotonic Critical Temperature in Superconductor/ Ferromagnet Bilayers, Phys. Rev. B 66, 014507 (2002).

[23] C. Cirillo, S. L. Prischepa, M. Salvato, C. Attanasio, M. Hesselberth, and J. Aarts, Superconducting Proximity Effect and Interface Transparency in $\mathrm{Nb} / \mathrm{PdNi}$ Bilayers, Phys. Rev. B 72, 144511 (2005).

[24] G. J. van Baarle, A. M. Troianovski, T. Nishizaki, P. H. Kes, and J. Aarts, Imaging of Vortex Configurations in Thin Films by Scanning-Tunneling Microscopy, Appl. Phys. Lett. 82, 1081 (2003).

[25] F. J. Jedema, B. J. van Wees, B. H. Hoving, A. T. Filip, and T. M. Klapwijk, Spin-Accumulation-Induced Resistance in Mesoscopic Ferromagnet-Superconductor Junctions, Phys. Rev. B 60, 16549 (1999). 